\title{
Large amplitude perturbations and waves at the duskside LLBL of the magnetopause generated by an interplanetary tangential discontinuity on December 7, 2000
}

\author{
F. T Gratton ${ }^{1}$, C. J. Farrugia ${ }^{2}$, L. Bilbao ${ }^{1}$, G. Gnavi ${ }^{1}$, and E . Lund ${ }^{2}$ \\ ${ }^{1}$ Instituto de Física del Plasma, CONICET-UBA, Argentina \\ ${ }^{2}$ Space Research Center, Univ. New Hampshire, NH, USA
}

\begin{abstract}
On December 7, 2000 a joint current sheeth and vorticity layer hit the magnetopause (MP) generating large amplitude oscillations, and wave-like perturbations observed by CLUSTER at the near dusk flank. Linear stability theory and MHD numerical simulations support the hypothesis that the waves were due to the Kelvin-Helmholtz (KH) instability. The simulation brings in light novel dynamic properties of the boundary layer under the KH excitation.
\end{abstract}

Keywords: magnetopause; Kelvin-Helmholtz instability; magnetospheric physics, MHD theory.

PACS: 94.30.ch; 94.30.cq; 94.30.-d; 94.05.-a.

\section{INTRODUCTION}

On December 7, 2000 an interplanetary tangential discontinuity (TD), at the same time an electric current and a vorticity layer, were identified in ACE data. ${ }^{[1]}$ The TD was followed by a lapse of small clock angle $\phi$ of the interplanetary magnetic field (IMF). After the TD impact on the magnetosphere, CLUSTER located at the near duskside, low latitude boundary layer of the magnetopause (MP) observed a burst of large amplitude oscillations, followed by wave-like perturbations during the low $\phi$ period. Based on CLUSTER data recorded in the large oscillations lapse, a local stability analysis of the MP supports the Kelvin-Helmholtz (KH) instability as the cause of the waves observed in the boundary layer. A study of the coupling of the MP perturbations with the excitation of ground magnetometer chains is in agreement with the KH conjecture: it shows evidence of magnetospheric resonant shells (with typical phase jumps) and seems to exclude the idea of global windsock motions of the MP. ${ }^{[1]}$ We show results of a $3 D+t$ nonlinear MHD simulation of the KH instability in a model MP based on parameters measured by CLUSTER. Novel features of the physical process that broadens and modifies the MP structure are revealed by the simulation and pointed out here.

\section{Interplanetary Plasma and Fields}

The December 7, 2000, event was characterized by a sequence of interplanetary directional discontinuities hitting the magnetosphere. CLUSTER spent several hours on an outbound crossing of the duskside boundary layer at $\sim$ 3:00 MLAT, in almost radial direction. The long duration came about because the MP was slowly expanding at the same time. The IMF rotated northward, but the change was interrupted by several abrupt east-west rotations of field and flow. We focus on one impulsive change, shown to be a TD by a minimum variance analysis of the $\boldsymbol{B}$ field recorded by ACE, from which the orientation and normal velocity of propagation was derived. ${ }^{[1]}$ Later, after an estimated time lag of $75 \mathrm{~min}$ to CLUSTER, two wave types were observed at the MP, during and after the TD impinged on it. Fig. 1 shows data from ACE on a short time scale to focus on the TD properties. Important variations of $V_{i}$ and $B_{i}$ can be seen $(i, j, k$ : local Cartesian triad on the TD plane with $k$ along the normal). In GSM these changes are mainly in the $V_{y}, B_{y}$, components. We point out that $P_{d y n}$ remained constant across the transition, and that the density change across the layer was negligible. The first condition ensures that the stand-off magnetopause point does not change on arrival of the TD. The significant variation was in the tangential stresses $P_{k i}=$ $\rho V_{i} V_{k}$, which are smaller but comparable with $P_{d y n}$; 
pressure components ratio $r=V_{i} / V_{k} \approx 1 / 3 . B_{i}$ has a minor effect on the ponderomotive force on the MP, but has influence on the geomagnetic field and the changes of the MP current sheath. Conclusion is that the observed TD was a joint current sheath - vorticity layer with uniform density. Fig. 2 shows a sketch of the TD, and a MP model (Shue et al., 1998) where CLUSTER position is also indicated.

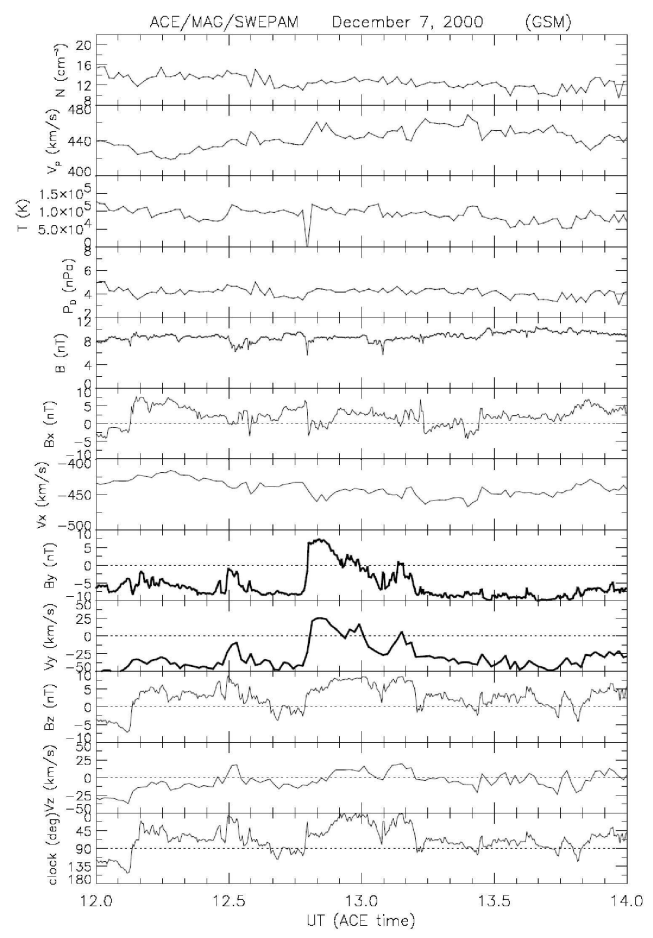

FIGURE 1. TD features identified in ACE's data; note the sudden change in $B_{y}, V_{y}$.

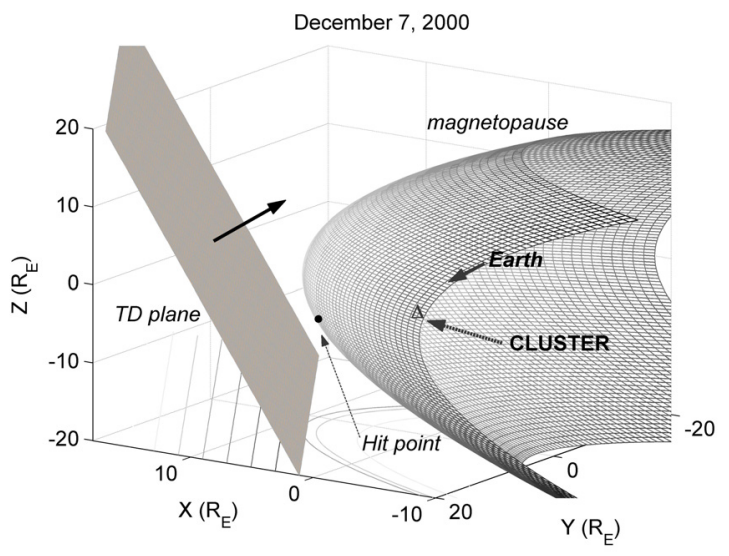

FIGURE 2. Schematic of the TD from ACE data, and the MP from Shue et al., model.

\section{Flank Observations}

Fig. 3 shows the large oscillations (prominent in $T$ and $n$ ) and the subsequent wavy perturbations (evident in the $\boldsymbol{B}$ panels) recorded by CLUSTER. These features start in close correlation with the TD observed by ACE taking into account the 75 min delay; superposed in the plots are also ACE data with the time shift. After $\approx 14: 00$ UT, plasma sheet and magnetosheath plasma intermixed, or alternating in three - four large oscillations, was observed (Fig. 3). From 14:10 to 14:25 UT, instead, waves with $\sim 75 \mathrm{~s}$ period $(13 \mathrm{mHz})$ appeared in coincidence with a change to nearly due north of the IMF. CLUSTER spectrograms (not shown) also indicate an intermittence of energetic $\mathrm{O}^{+}$and $\mathrm{H}^{+}$ions of magnetospheric origin, well correlated with the quasiperiodic $\boldsymbol{B}$ perturbations. ${ }^{[1]}$

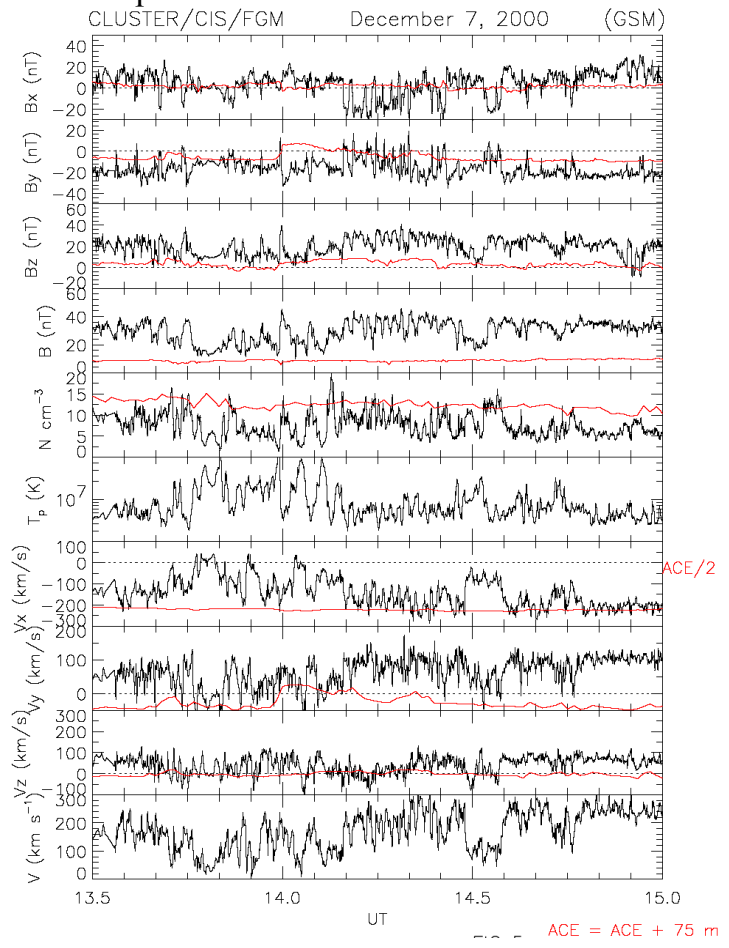

FIGURE 3. Perturbations observed by CLUSTER: large amplitude oscillations and waves.

\section{The KH Hypothesis}

A local stability analysis of a MP part (around CLUSTER position) against perturbations of the $\mathrm{KH}$ type was done using a linear compressible MHD code. A description would be too long to fit here, for equations and symbols see these Proceedings ${ }^{[2]}$, and a derivation in ref.3. The model is a plane slab, uniform over local coordinates $X, Z$, and stratified along $Y$ (normal to the MP). The boundary value problem for 
the KH instability is solved by a shooting method. The field profiles in $Y$ are hyperbolic tangents that join asymptotic quantities on both sides of the transition (indices 1,2), obtained reading data values at the extremes of the large oscillations observed by CLUSTER after $\approx 14: 00$ UT. The input parameters chosen are in Table 1. Fig. 4 gives the growth rate $g \equiv \gamma d / U_{1} \quad v s$ the wave number $k d$, both as non dimensional quantities; $d=\Delta / 2$ is half the velocity

TABLE 1. Parameters for Dec. 7, 2000.

\begin{tabular}{|l|l|l|l|}
\hline $14: 05 \mathrm{UT}$ & magnetosh. & $14: 03 \mathrm{UT}$ & magnetosph. \\
\hline$n_{1}$ & $13.6 \mathrm{~cm}^{-3}$ & $n_{2}$ & $2.3 \mathrm{~cm}^{-3}$ \\
\hline$B_{1}$ & $34.6 \mathrm{nT}$ & $B_{2}$ & $22.1 \mathrm{nT}$ \\
\hline$U_{l}$ & $139 \mathrm{~km} / \mathrm{s}$ & $U_{2}$ & $7.9 \mathrm{~km} / \mathrm{s}$ \\
\hline$T_{l}$ & $0.378 \mathrm{kev}$ & $T_{2}$ & $\sim 4.07 \mathrm{kev}$ \\
\hline$\theta_{l}\left(B_{I} V\right)$ & $121^{\circ}$ & $\theta\left(B_{2} V\right)$ & $126^{\circ}$ \\
\hline
\end{tabular}

gradient scale ( $\Delta \sim$ LLBL thickness) and $U_{l}$ is the magnetosheath velocity. The result is for the angle $\varphi=32^{\circ}$ between $\boldsymbol{k}$ and $\boldsymbol{V}$, found to be the most favorable for the growth of the instability. The modes are stabilized by shifting the $\boldsymbol{k}$ direction $\sim \pm 10^{\circ}$ from $\varphi=32^{\circ}$ (or $\varphi=32^{\circ}+180^{\circ}$ ). Therefore, the instability develops with a distinct $\boldsymbol{k}$ orientation.

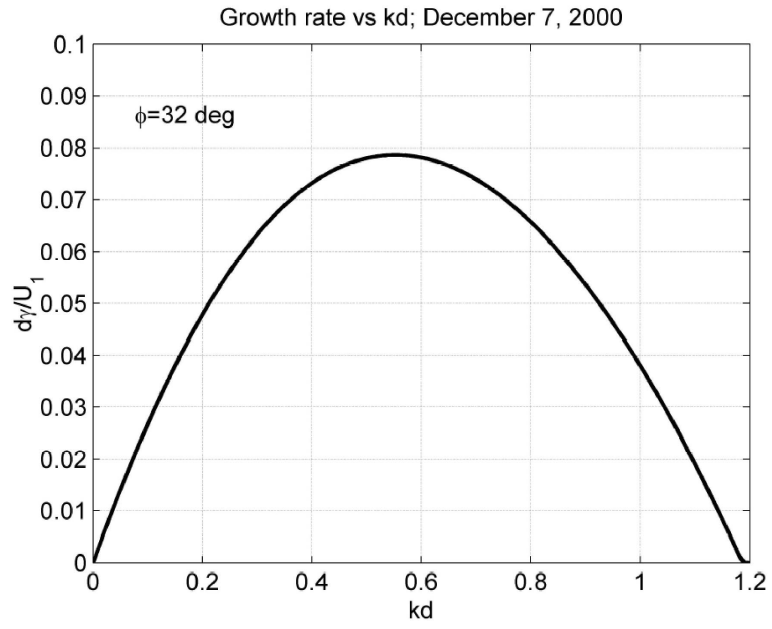

FIGURE 4. Normalized growth rate of the KH instability as a function of $k d$ (one scale model).

At the CLUSTER site, $M=0.57, M_{A}=0.70$ (sound and Alfvénic Mach numbers, respectively) and the local magnetic shear angle $d \theta$ was found to be very small, $\sim 5^{\circ}$. The maximum growth rate occurs for $\lambda \approx 5.7 \Delta$ with an e-folding time $\tau_{e} \approx 146 \mathrm{~s}$ if we assume $\Delta=0.5 R_{E}$. Compressibility scales with $M^{2}$ and its effects are present but not dominant. However, $M_{A}<1$ so that the magnetic tensions are strong, and the most unstable mode has $\boldsymbol{k} \approx$ perpendicular to the average directions of the magnetosheath and magnetospheric fields. We conclude that the lessening of the IMF clock angle $\phi$ (during the interval $\approx 14: 03$ - 14:08 UT), which became smaller than $\sim 22.5^{\circ}$ and stayed small during the next ten minutes, was favorable to the $\mathrm{KH}$ instability in general (see ref.4) and in particular in the dusk flank, at the CLUSTER locale, reduced $d \theta$ substantially. Thus, the theory predicts that the MP was $\mathrm{KH}$ unstable at the CLUSTER site. The $\mathrm{KH}$ interpretation is also supported by a study of the response of ground magnetometers ${ }^{[1]}$ mentioned in the introduction, which we have no space to report on here. However, the wave-like perturbations appeared very soon after the fall of $\phi$ : if they were of KH nature we must assume that the preceeding large oscillations caused a drop in the amplification time. The MP perturbation generated by TD hit accelerated the $\mathrm{KH}$ process.

\section{A non linear simulation of the $\mathrm{KH}$ instability}

We confirmed the idea of a fast evolution with a nonlinear simulation of the $\mathrm{KH}$ instability. A finite volume, arbitrary Lagrangian-Eulerian, $3 \mathrm{D}+\mathrm{t}$, code for compressible ideal MHD flows was written by L. Bilbao to study the nonlinear evolution of unstable perturbations in plane-slab models of the MP with input parameters suggested by spacecraft data. For the main concepts and code features see ref.5. The initial structure is the same laminar current-sheet and vorticity layer used in the linear stability analysis of the MP. In the code run we report the initial random excitation was set in the velocity field at $\sim 20 \%$ level, to simulate a strong perturbation of the flow due to the TD hit, and the perturbation pattern was oriented with the $\varphi=32^{\circ}$ angle, indicated as the mode of fastest growth. For the computational box periodic conditions on the $X, Z$, faces were assumed, while fixed flow and fields were imposed on the $Y$ boundaries.

Fig. 5 shows the distribution of vorticity, $\Omega \equiv \omega \times \operatorname{sign}\left(\omega_{Z}\right)(\omega=|\operatorname{curl}(\boldsymbol{V})|)$ at $t=180 \mathrm{~s}$ after the start (axes are in Earth radii). Three $Z$ slices and the $X$ back side show vorticity contours. A few streamlines are also indicated, three sets of three lines each to illustrate the velocity field. From an initial positive $\Omega$ planar sheet, a distorted configuration with much larger but comparable positive and negative $\Omega$ values has developed. The plot shows also accumulation of large and opposite rotation in core pairs. The vorticity pattern grows aligned with the magnetic field lines, which are shown in Fig. 6 at $\mathrm{t}=160 \mathrm{~s}$, together with temperature contours. We find high temperatures $(3 \times$ magnetospheric value) and low density in the pair of counter vortices. A considerable broadening of the original structure is apparent in both plots. Fig. 7 illustrates the mixing of magnetosheath and 
magnetospheric plasma at $t=120 \mathrm{~s}$, emphasized by a plot of the surface of constant density $n=9 \mathrm{~cm}^{-3}$ (a midway value between $n_{1}, n_{2}$ ) that shows a typical rollover effect. On the isodensity surface the velocity field is indicated by cones.

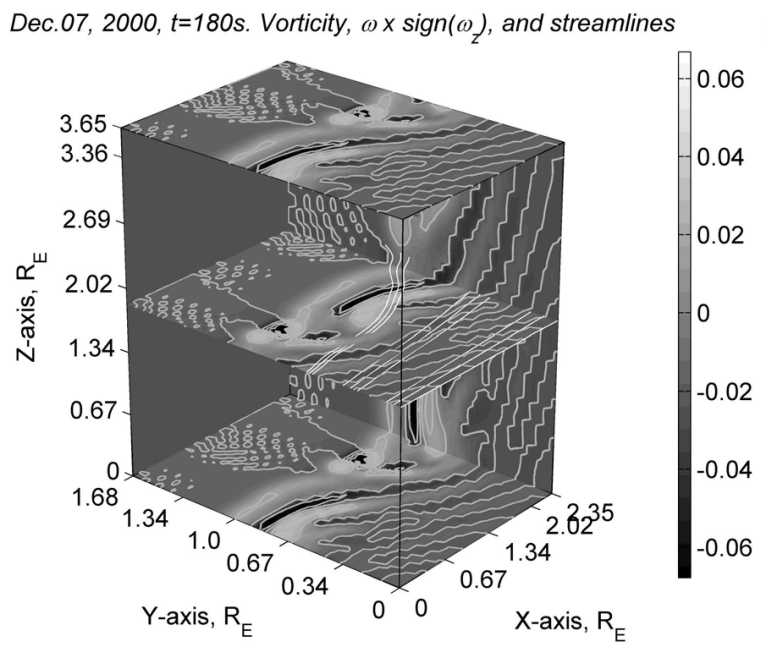

FIGURE 5. Vorticity contours and a sample of three sets of streamlines at $\mathrm{t}=180 \mathrm{~s}$.

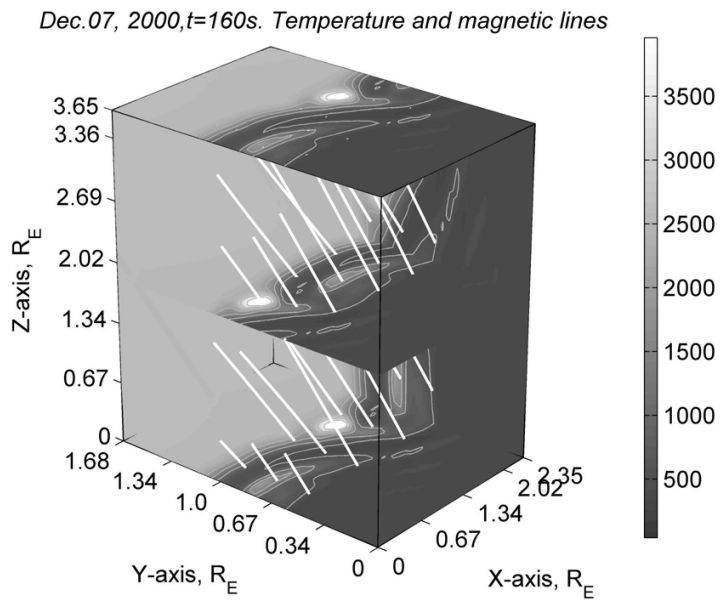

FIGURE 6. Temperature and magnetic lines, at $\mathrm{t}=160 \mathrm{~s}$.

The results can be summarized as follows. i) The rise time of large non linear features is $\leq \tau_{e}$ when the initial structure is already significantly perturbed: "acceleration of the instability". ii) Formation of counter-rotating cores with $\omega$ larger than the initial vorticity strength. iii) The plasma in the pair of vortex cores is hot $\left(\sim 3 \times T_{2}\right)$ and tenuous $\left(\sim 1 / 3 \times n_{2}\right)$. iv) Broadening of the boundary layer and plasma mixing, producing configurations favorable to enhanced particle diffusion. v) "Rigidity" of the magnetic field lines (for the $M_{A}=0.7$ value explored) apparent in the strong influence of the magnetic field direction on the nonlinear dynamics. However, the process is not entirely two-dimensional, bona fide 3-D features are also present.

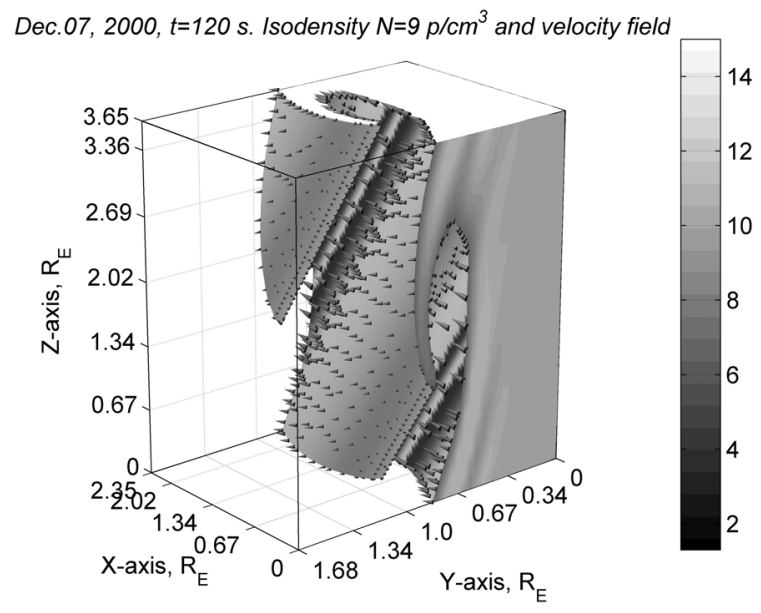

Figure 7. Surface of equal density $n=9 \mathrm{p} / \mathrm{cm}^{3}$ at $\mathrm{t}=120 \mathrm{~s}$ with cones that represent the velocity field.

\section{ACKNOWLEDGMENTS}

Research supported by Argentinean grants: CONICET PIP 5291, and UBACYT X291. USA, NASA grants: NAG5-13116, NAG5-12189, and NNG05GG25G. Bilbao, Gratton, and Gnavi, are members of the Consejo Nacional de Investigaciones Científicas y Técnicas (CONICET).

\section{REFERENCES}

1. Farrugia, C. J., et al., EOS Trans. AGU, 86 (18), Jt. Assem. Suppl., 2005.

2. Gnavi, G., F. T. Gratton, C. J. Farrugia , and L. E. Bilbao, 2005, these Proceedings.

3. Gratton, J., A. G. González, and F. T. Gratton, Plasma Phys. \& Contr. Fusion, 30, 435, 1988.

4. Farrugia, C. J., F. T. Gratton, L. Bender, et al., J. Geoph. Res., 103, 6703, 1998

5. Bilbao, L. E., 2005, these Proceedings. 